\title{
Underutilization of Portable Orders for Life-Sustaining Treatment at Discharge from Hospital: Observational Study at US Academic Trauma Center
}

\author{
Jeffrey B. Rubins, $M D^{1,2}$ \\ 'University of Minnesota, Minneapolis, MN, USA; ${ }^{2}$ Palliative Care Division, Hennepin Healthcare, Minneapolis, MN, USA.
}

\begin{abstract}
BACKGROUND: Decisions to limit use of life-sustaining treatment occur frequently during hospitalizations, and portable medical orders (also known as Portable Orders for Life-Sustaining Treatment (POLST)) can ensure that patient preferences regarding resuscitation are followed after discharge.

OBJECTIVE: To determine the frequency and predictors of completion of POLST orders for adults with change during hospitalization in resuscitation status to Do Not Resuscitate.

DESIGN: Retrospective observational study at level 1 trauma and academic hospital in Minneapolis, MN, USA PARTICIPANTS: All adults (18 years or older) hospitalized between June 2017 and June 2018, inclusive, with code status changed from Full Code to DNR. For patients with more than one hospitalization during this study interval, only the first hospitalization when DNR was ordered was included in this analysis.
\end{abstract}

MAIN MEASURES: Completion of POLST orders by time of discharge.

KEY RESULTS: From 2017 to 2018, 160 adults had a change from Full Code to DNR status during index hospitalization and survived to discharge, most (156 patients) to a nursing care facility. Of these, only 50 (31.2\%) had POLST orders provided at discharge. Documentation of informed refusal of intubation in addition to DNR status was a significant predictor (OR 4.1, 99\% CI 1.5-11.0) of POLST orders on discharge, as was admission to a medical service compared with non-medical service (OR 3.2, 99\% CI 1.1-12.2). Palliative care consultants, rather than primary providers on the hospital services, completed most POLST orders.

CONCLUSIONS: Despite primary hospital providers engaging in conversations regarding resuscitation and entering DNR orders during hospitalization, the majority of patients in our study discharged to other care facilities without POLST orders. POLST orders are a simple palliative care tool available to primary hospital providers to help ensure continuity of plan of care at discharge for patients who wish to avoid invasive life-sustaining treatments and/or cardiopulmonary resuscitation.

KEY WORDS: advance care planning; portable medical orders; care transitions; DNR; Physician Orders for Life-Sustaining Treatment.

Prior Presentation None

Received October 2, 2019

Accepted January 31, 2020

Published online February 10, 2020
J Gen Intern Med 35(7):2065-8

DOI: $10.1007 / \mathrm{s} 11606-020-05698-1$

(c) Society of General Internal Medicine 2020

\section{INTRODUCTION}

In the early 1990s, Oregon developed the Physician Orders for Life-Sustaining Treatment (POLST) as a set of portable medical orders, intended to translate a patient's end-of-life treatment preferences into actionable medical orders. ${ }^{1}$ The National POLST Paradigm established in 2018 quality standards for POLST orders and programs, currently referred to by a variety of acronyms (Table 1), and assists 46 states with active or developing POLST programs. Although the National POLST Paradigm recommends the term "portable medical orders," we will use the most familiar term "POLST" orders in this manuscript.

Decisions to limit use of life-sustaining treatment occur frequently during hospitalizations, and the intent of POLST orders is to ensure continuity of the plan of care across healthcare settings by communicating patient's wishes about resuscitation and aggressiveness of care. Most patients (and some providers) are not aware that despite documentation of a patient's wishes in healthcare directives, provider's notes, and/ or hospital orders for Do Not Resuscitate, emergency medical responders are required to initiate full resuscitative efforts for out of hospital cardiopulmonary arrest unless POLST orders are available and indicate wishes to forego these treatments. ${ }^{2}$ Consequently, patients with serious illness who have POLST orders are significantly more likely to have their end-of-life wishes honored than those with advance directives only. 2,3 Therefore, completion of POLST orders at hospital discharge is recommended for patients who wish to forego resuscitation to extend this treatment plan during transport and beyond the hospitalization. ${ }^{1,4-6}$ Studies to date suggest that POLST orders, when available, are associated with patients receiving treatments they desire in hospitals and nursing facilities, and spending their end of life in their preferred location. ${ }^{2,7-10}$

However, there are little data regarding how often POLST orders are completed for hospitalized patients who have changed their treatment preferences from Full Code to Do Not Resuscitate (DNR) prior to discharge. One recent study reported only $38 \%$ completion of POLST orders before 
Table 1 POLST Paradigm Forms by State

\begin{tabular}{|c|c|}
\hline Form & State(s) \\
\hline $\begin{array}{l}\text { POLST (Physician Orders for } \\
\text { Life-Sustaining Treatment) }\end{array}$ & 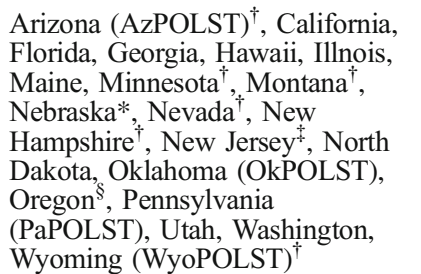 \\
\hline $\begin{array}{l}\text { MOST (Medical Orders for } \\
\text { Scope of Treatment) }\end{array}$ & $\begin{array}{l}\text { Alaska, Colorado, Delaware } \\
\text { (DMOST), New Mexico, North } \\
\text { Carolina, Texas }\end{array}$ \\
\hline $\begin{array}{l}\text { POST (Physician Orders for } \\
\text { Scope of Treatment) }\end{array}$ & $\begin{array}{l}\text { Idaho, Indiana, Iowa (IPOST), } \\
\text { Louisiana (LaPOST), Mississippi*, } \\
\text { South Carolina, Tennessee, } \\
\text { Virginia, West Virginia }\end{array}$ \\
\hline $\begin{array}{l}\text { TPOPP (Transportable Physician } \\
\text { Orders for Patient Preferences) }\end{array}$ & Kansas, Missouri \\
\hline $\begin{array}{l}\text { MOLST (Medical Orders for } \\
\text { Life-Sustaining Treatment) }\end{array}$ & $\begin{array}{l}\text { Connecticut*, Maryland*, } \\
\text { Massachusetts, New York, Ohio, } \\
\text { Rhode Island }\end{array}$ \\
\hline $\begin{array}{l}\text { COLST (Clinician Orders for } \\
\text { Life-Sustaining Treatment }\end{array}$ & Vermont* \\
\hline
\end{tabular}

hospital discharge for patients with DNR orders, but did not specify whether the DNR status was a change during that hospitalization. ${ }^{6}$ Therefore, to add to understanding of this important transition of care, we studied how frequently providers completed POLST orders for hospitalized patients with change from Full Code to DNR status during hospitalization, and predictors of whether POLST orders were provided at discharge.

\section{METHODS}

We conducted a retrospective, observational study including all patients 18 years and older with DNR orders placed during hospitalization between June 2017 and June 2018 at Hennepin County Medical Center (HCMC, Hennepin Healthcare, Minneapolis, MN), a nationally recognized 440-bed level 1 trauma center, academic training hospital, and safety-net hospital with over 20,000 adults admitted for acute care each year. The Institutional Review Board of the Minnesota Medical Research Foundation/Hennepin Healthcare approved this study and waived the requirement for informed consent.

\section{Data Sources}

A total of 422 cases were identified by a search of the electronic health record (Epic Systems Corporation, Madison, WI) for adults admitted during the defined study interval with DNR orders placed during hospitalization. For patients with more than one hospitalization during this study interval, the first hospitalization when DNR was ordered was reviewed. Data from POLST order forms, which are scanned into Epic when present on admission and/or discharge, were manually extracted from scanned documents.

\section{Outcomes}

The predetermined outcome was evidence of POLST orders written prior to discharge for patients with a change from Full Code to DNR during hospitalization.

\section{Statistical Analysis}

We used Stata software (version 15.1; StataCorp) for all analyses. For comparisons of binary outcomes, we used logistic regression analysis and reported odds ratio and confidence intervals. For comparison of continuous variables, we reported means and standard errors (SE). To reduce risk of type I error from multiple comparisons, our predetermined alpha was set at 0.01 .

\section{RESULTS}

Of 422 total hospitalized adults with DNR orders during the 12-month study period, 255 had DNR orders placed at time of admission (160 with POLST form available at time of admission indicating DNR, the remainder based on other documentation of DNR status or discussion of DNR status). The remaining 167 patients had orders placed for "Full Code" at time of admission (hospital day 1).

Of the 167 patients who had a change in their resuscitation status from Full Code to DNR, 160 (95.8\%) survived to discharge. Of these 160 patients, $50(31 \%)$ had POLST orders provided at discharge indicating DNR status, signed on average 2.5 days before discharge (99\% CI 1.1-3.9). Compared with patients without POLST orders, completion of POLST orders on discharge was not significantly associated with patient age, male gender, documentation of a legal surrogate (guardian or healthcare agent/power of attorney (POA) for healthcare), or length of hospitalization (Table 2). Only four of 160 patients (2.5\%) discharged to independent living, none with completion of POLST orders on discharge; the remainder were discharged to acute/subacute rehabilitation, to long-term acute care hospital, or to long-term nursing care, and completion of POLST orders at discharge was not associated with discharge location (OR 0.99, 99\% CI 0.74-1.33).

Completion of POLST orders on discharge was significantly associated with documentation of discussion and informed refusal of intubation (noted in 38 of the 50 patients), and was significantly higher for patients admitted to a medical hospital service (Family Medicine, Hospitalist, or Medicine/House staff) compared with admission to a non-medical hospital service (Intensive Care Unit, Neurology, Psychiatry, Surgery) (Table 2). However, rates of POLST order completion were low even on medical services, documented for 23 of 66 (35\%) of Medicine/House staff, 15 of 46 (33\%) of Hospitalist, and seven of 14 (50\%) of Family Medicine patients (differences not statistically significant). 
Table 2 Association of Patient- and Hospital-Related Factors with Completion of POLST Orders at Discharge

\begin{tabular}{llllllll}
\hline \hline POLST orders & $\begin{array}{l}\text { Total } \\
\text { No. (\%) }\end{array}$ & $\begin{array}{l}\text { Age, years } \\
\text { Mean (SE) }\end{array}$ & $\begin{array}{l}\text { Male } \\
\text { No. (\%) }\end{array}$ & $\begin{array}{l}\text { Legal surrogate } \\
\text { No. (\%) }\end{array}$ & $\begin{array}{l}\text { LOS }^{\mathbf{b}} \text {, days } \\
\text { Mean (SE) }\end{array}$ & $\begin{array}{l}\text { Refused intubation }^{\mathbf{c}} \\
\text { No. (\%) }\end{array}$ & $\begin{array}{l}\text { Medical services }^{\mathbf{d}} \\
\text { No. (\%) }\end{array}$ \\
\hline Yes & $50(31)$ & $74.7(1.8)$ & $25(50.0)$ & $16(32.0)$ & $15.5(1.7)$ & $38(76.0)$ & $5(10.0)$ \\
No & $110(69)$ & $78.0(1.2)$ & $47(42.7)$ & $40(36.4)$ & $11.4(1.5)$ & $48(43.6)$ & $29(26.4)$ \\
Total & $160(100)$ & $77.0(1.0)$ & $72(45.0)$ & $56(35.0)$ & $12.7(1.2)$ & $86(53.7)$ & $34(21.1)$ \\
OR $^{\mathbf{a}}$ & & 0.98 & 1.34 & 0.82 & 1.02 & 4.10 & 3.22 \\
$99 \%$ CI & & $0.95-1.01$ & $0.56-3.24$ & $0.32-2.09$ & $0.99-1.04$ & $1.52-11.0$ & $1.1-12.2$ \\
\hline
\end{tabular}

${ }^{a}$ Odds of POLST orders provided at discharge compared with control (no POLST)

${ }^{b}$ Hospital length of stay

${ }^{c}$ Documentation of patient's informed refusal of intubation in progress notes or orders

${ }^{d}$ Medical hospital services (Family Medicine, Hospitalist, Medicine) compared with non-medical ward services (Intensive Care Unit, Neurology,

Psychiatry, Surgery)

Of the 110 patients with change in code status to DNR but without POLST orders at discharge, the majority (73 patients) did not have any documentation of discussion of POLST orders in their medical records. Documentation for the other 37 patients without POLST orders at discharge indicated the following: (1) plan for the primary team or hospice team to complete POLST orders (11 patients); (2) evidence of POLST orders within the previous year, but without indication of a plan to provide updated POLST after index hospitalization (21 patients); (3) documentation that no POLST order was available, without indication of plan to provide POLST orders (four patients); (4) documentation of discussion of POLST orders with patient, but patient and family declining to have POLST orders on discharge (one patient).

Palliative care was consulted for 82 of the 160 patients (51.3\%), and 47 of these 82 patients had POLST orders at discharge $(57.3 \%$ of consults, and $94 \%$ of all patients with POLST orders at discharge). Most of the POLST orders were completed by palliative care consultants rather than primary teams: 11 of 23 (48\%) on Medicine/House staff service, 13 of $15(87 \%)$ on Hospitalist service, six of seven (86\%) on Family Medicine, and all of the POLST orders on non-medical ward services. To elucidate why a sizable minority of patients with palliative care consults did not have POLST orders on discharge, we compared those with (47 patients) and without (35 patients) POLST orders. POLST orders at discharge were associated with consults for "goals of care" rather than symptom management (OR 11.5, 95\% CI 1.34-98.5), consult service follow-up with patient until day of discharge (OR 27.18, 99\% CI 1.7-427.4), and discharge with hospice services (OR $3.5,99 \%$ CI 1.04-11.85).

\section{DISCUSSION}

The intent of POLST orders is to ensure continuity of the plan of care across healthcare settings by communicating patient wishes about resuscitation and aggressiveness of care. When discussions and informed decisions about foregoing resuscitation are made during a hospitalization, missing the opportunity to provide POLST orders at hospital discharge may lead to subsequent end-of-life care that is inconsistent with patients' goals, including undesired out of hospital resuscitation and death in the hospital. ${ }^{2,-10}$ Some states have emphasized the importance of providers completing POLST orders at discharge by legally mandating completion of POLST forms. ${ }^{11}$

To our knowledge, our study is the first to assess the rates and predictors of POLST order completion on discharge of patients who had resuscitation status changed from Full Code to DNR during hospitalization. Most of our patients (95.8\%) who had resuscitation status changed to DNR during hospitalization survived to discharge, and without POLST orders were at risk for undesired attempts at resuscitation if they suffered cardiac arrest during transport or upon arrival at their discharge location. However, only $32.3 \%$ of these patients had completion of POLST orders at discharge, similar to a recently published incidence of $38 \%$ for patients with DNR status in a study by Haynes et al. ${ }^{6}$; of note, their study did not specify that patients were Full Code status on admission.

We did not find a significant association between completion of POLST orders on discharge and patient age, gender, documentation of a legal surrogate, length of hospitalization, or discharge location. Patient refusal to have POLST orders (with the exception of one patient) did not explain discharge without POLST orders. However, completion of POLST orders on discharge was significantly associated with documentation of discussion and informed refusal of intubation, and with a medical (as opposed to non-medical) service providing inpatient care, with highest rates of POLST order completion on Medicine/House staff, Hospitalist, and Family Medicine teams. Importantly, palliative care consultants rather than the primary team completed most of the POLST orders on the medical services, and all of the POLST orders on the nonmedical services. However, involvement of palliative care consultants did not ensure completion of POLST orders on discharge from these hospital services, especially when consult requests were for symptom management, when the consult team did not follow the patient up until discharge, or when patients discharged with hospice services.

Our results underscore the need to have an established process for completion of POLST orders at hospital discharge for patients who desire to limit use of cardiopulmonary resuscitation and life-sustaining treatments. We speculate that primary hospital providers, despite being comfortable discussing 
patient's preferences regarding CPR and writing DNR orders, may feel that they lack the time, experience, access to forms, and understanding of the importance of POLST orders to the post-hospital discharge plan of care, and therefore do not complete POLST orders. Although arguments have been made for having primary care providers or primary specialists (e.g., oncologists) take responsibility for completion of POLST orders, ${ }^{12}$ these providers are rarely part of the primary care team when patients are hospitalized. As we noted in this study, palliative care consultants provided the large majority of POLST orders at discharge, but they often are not part of the discharge process. Given provisions now available for reimbursement from Medicare for advance care planning, ${ }^{13}$ and the emphasis on providers having primary palliative care skills, ${ }^{14}$ ${ }^{17}$ our findings would support investment in training of hospital providers in the use POLST orders as part of the discharge process, which in turn can help to accomplish healthcare goals of improving care coordination and reducing readmissions. ${ }^{18}$ Looking forward, health informatics solutions to assist providers with POLST orders (which have already been implemented in some states, including development of electronic POLST registries in New York, Idaho, Oregon, Utah, and West Virginia) and integration of POLST completion into electronic health records ${ }^{19}$ will likely help normalize the process of providing POLST orders at discharge.

Acknowledgments: We thank Dr. David Rubins for his critical review and revision of this manuscript.

Corresponding Author: Jeffrey B. Rubins, MD; Palliative Care Division, Hennepin Healthcare, 701 Park Ave G5, Minneapolis, MN 55415, USA (e-mail: Jeffrey.Rubins@hcmed.org).

\section{Compliance with Ethical Standards:}

The Institutional Review Board of the Minnesota Medical Research Foundation/Hennepin Healthcare approved this study and waived the requirement for informed consent.

Conflict of Interest: The author has no real or perceived conflict of interest with the material presented in this article.

\section{REFERENCES}

1. National POLST Paradigm. Available at: https://polst.org. Accessed December 8, 2019.
2. Richardson DK, Fromme E, Zive D, Fu R, Newgard CD. Concordance of out-of-hospital and emergency department cardiac arrest resuscitation with documented end-of-life choices in Oregon. Ann Emerg Med 2014;63:375-383.

3. Pedraza SL, Culp S, Knestrick M, Falkenstine E, Moss AH. Association of physician orders for life-sustaining treatment form use with end-of-life care quality metrics in patients with cancer. JOP 2017;13: e881-e888.

4. Meier DE, Beresford L. POLST offers next stage in honoring patient preferences. J Palliat Med 2009;12:291-5.

5. Hickman SE, Nelson CA, Smith-Howell E, Hammes BJ. Use of the Physician Orders for Life-Sustaining Treatment program for patients being discharged from the hospital to the nursing facility. J Palliat Med 2014;17:43-9.

6. Haynes CA, Shiell-Earp CN, Wenger NS, Simon WM, Skootsky SA, Clarke R, et al. Improving communication about resuscitation preference for patients discharged from hospital to nursing home: a quality improvement project. J Palliat Med 2019;22:557-560.

7. Hopping-Winn J, Mullin J, March L, Caughey M, Stern M, Jarvie J. The progression of end-of-life wishes and concordance with end-of-life care. J Palliat Med 2018;21:541-5.

8. Fromme EK, Zive D, Schmidt TA, Cook JN, Tolle SW. Association between Physician Orders for Life-Sustaining Treatment for Scope of Treatment and in-hospital death in Oregon. $J$ Am Geriatr Soc 2014;62:1246-51.

9. Hickman SE, Nelson CA, Moss AH, Tolle SW, Perrin NA, Hammes BJ. The consistency between treatments provided to nursing facility residents and orders on the physician orders for life-sustaining treatment form. $J$ Am Geriatr Soc 2011;59:2091-9.

10. Collier J, Kelsberg G, Safranek S. Clinical Inquiries: How well do POLST forms assure that patients get the end-of-life care they requested? J Fam Pract 2018;67:249-51.

11. Maryland Medical orders for life-sustaining treatment (molst) form-health general articles §§ 5-608.1, 5-608, 5-609, and 5-617. 2013.

12. Tolle SW, Back AL, Meier DE. Clinical decisions. End-of-life advance directive. N Engl J Med 2015;372:667-70.

13. Pope TM. Legal briefing: Medicare coverage of advance care planning. $J$ Clin Ethics 2015;26:361-7.

14. Quill TE, Abernethy AP. Generalist plus specialist palliative carecreating a more sustainable model. N Engl J Med 2013;368:1173-5.

15. Weissman DE, Meier DE. Identifying patients in need of a palliative care assessment in the hospital setting: a consensus report from the Center to Advance Palliative Care. J Palliat Med 2011;14:17-23.

16. Carroll T, El-Sourady M, Karlekar M, Richeson A. Primary palliative care education programs: review and characterization. Am J Hosp Palliat Care 2019;36:546-9.

17. Schenker Y, Quill TE. Primary palliative care. UpToDate 2018. Accessed 11-29-2019.

18. Kripalani S, Theobald CN, Anctil B, Vasilevskis EE. Reducing hospital readmission rates: current strategies and future directions. Annu Rev Med 2014;65:471-85.

19. Zive DM, Cook J, Yang C, Sibell D, Tolle SW, Lieberman M. Implementation of a novel electronic health record-embedded physician orders for life-sustaining treatment system. J Med Syst 2016; 40:245.

Publisher's Note Springer Nature remains neutral with regard to jurisdictional claims in published maps and institutional affiliations. 